Research Article

\title{
On Refined Neutrosophic Matrices and Their Application in Refined Neutrosophic Algebraic Equations
}

\author{
Mohammad Abobala \\ Faculty of Science, Department of Mathematics, Tishreen University, Latakia, Syria \\ Correspondence should be addressed to Mohammad Abobala; mohammadabobala777@gmail.com
}

Received 7 January 2021; Accepted 1 February 2021; Published 13 February 2021

Academic Editor: Parimala Mani

Copyright (c) 2021 Mohammad Abobala. This is an open access article distributed under the Creative Commons Attribution License, which permits unrestricted use, distribution, and reproduction in any medium, provided the original work is properly cited.

The objective of this paper is to introduce the concept of refined neutrosophic matrices as matrices such as multiplication, addition, and ring property. Also, it determines the necessary and sufficient condition for the invertibility of these matrices with respect to multiplication. On the contrary, nilpotency and idempotency properties will be discussed.

\section{Introduction}

Neutrosophy is a new branch of generalized logic found by Smarandache to deal with indeterminacy in all fields of human knowledge. Neutrosophic sets were applicable in decision-making [1], number theory $[2,3]$, and space theory $[4,5]$.

The concept of refined neutrosophic structure was supposed firstly in [6] by splitting indeterminacy I into two levels of subindeterminacies $I_{1}$ and $I_{2}$. This idea was used in the study of refined neutrosophic rings [7-9], modules $[10,11]$, and groups [6]. Recently, the concept of $n$-refined neutrosophic structures was defined and used in [12-14].

Neutrosophic matrices were a useful tool to deal with indeterminacy in many studies; we find their basic definition and properties such as ring structure, multiplication, and other applications in $[15,16]$.

Through this work, we define, for the first time, the concept of refined neutrosophic matrices as a direct application of the refined neutrosophic set. Also, we determine the necessary and sufficient condition for the invertibility of these matrices with many related examples. On the contrary, we build an example to show how refined matrices can be used in refined neutrosophic equations defined in [17].

All refined neutrosophic matrices through this paper are defined over a neutrosophic field $F\left(I_{1}, I_{2}\right)$.

The structure of refined neutrosophic numbers is taken as $a+b I_{1}+c I_{2}$ instead of $\left(a, b I_{1}, c I_{2}\right)$. This representation is based on the theory of $n$-refined neutrosophic rings proposed in [12], where refined neutrosophic numbers can be represented by this form without any loss of generality or algebraic properties.

\section{Preliminaries}

Definition 1 (see [7]). Let $K$ be a field, the neutrosophic field generated by $K \cup I$, which is denoted by $K(I)=K \cup I$.

Definition 2 (see [7]). Classical neutrosophic number has the form $a+b I$, where $a$ and $b$ are real or complex numbers and $I$ is the indeterminacy such that $0 \cdot I=0$ and $I^{2}=I$ which results in $I^{n}=I$ for all positive integers $n$.

Definition 3 (neutrosophic matrix; see [15]). Let $M_{m \times n}=$ $\left\{\left(a_{i j}\right): a_{i j} \in K(I)\right\}$, where $K(I)$ is a neutrosophic field. We refer this to be the neutrosophic matrix.

Remark 1 (see [6]). The element $I$ can be split into two indeterminacies $I_{1}$ and $I_{2}$ with conditions

$$
\begin{aligned}
I_{1}^{2} & =I_{1}, \\
I_{2}^{2} & =I_{2}, \\
I_{1} I_{2} & =I_{2} I_{1}=I_{1} .
\end{aligned}
$$


Definition 4 (see [1]). If $X$ is a set, then $X\left(I_{1}, I_{2}\right)=\left\{\left(a, b I_{1}, c I_{2}\right): a, b, c \in X\right\}$ is called the refined neutrosophic set generated by $X, I_{1}$, and $I_{2}$.

Definition 5 (see [7]). Let $(R,+, \times)$ be a ring; $\left(R\left(I_{1}, I_{2}\right),+, \times\right)$ is called a refined neutrosophic ring generated by $R$, $I_{1}$, and $I_{2}$.

Theorem 1 (see [7]). Let $\left(R\left(I_{1}, I_{2}\right),+, \times\right)$ be a refined neutrosophic ring; then, it is a ring.

It is called a neutrosophic field if $R$ is a classical field.

Theorem 2 (see [17]). Let $A_{1} X_{1}+\cdots+A_{n} X_{n}=C$, $C=\left(c_{0}, c_{1} I_{1}, c_{2} I_{2}\right), X_{i}=\left(x_{0}^{(i)}, x_{1}^{(i)} I_{1}, x_{2}^{(i)} I_{2}\right)$, and $A_{i}=\left(a_{0}^{(i)}\right.$, $\left.a_{1}^{(i)} I_{1}, a_{2}^{(i)} I_{2}\right)$, be a linear equation with $n$-variables over $a$ refined neutrosophic field $F\left(I_{1}, I_{2}\right)$. Then, it is equivalent to the following system of classical linear equations over the classical field $F$ :
(a) $\sum_{i=1}^{n} a_{0}^{(i)} x_{0}^{(i)}=c_{0}$
(b) $\sum_{i=1}^{n}\left(a_{0}^{(i)}+a_{2}^{(i)}\right)\left(x_{0}^{(i)}+x_{2}^{(i)}\right)=c_{0}+c_{2}$
(c) $\sum_{i=1}^{n}\left(a_{0}^{(i)}+a_{1}^{(i)}+a_{2}^{(i)}\right)\left(x_{0}^{(i)}+x_{1}^{(i)}+x_{2}^{(i)}\right)=c_{0}+c_{1}+c_{2}$

\section{Main Concepts}

Definition 6 (refined neutrosophic matrix)
Let $A=\left(\begin{array}{ccc}a_{11} & \ldots & a_{1 m} \\ \vdots & \ddots & \vdots \\ a_{n 1} & \ldots & a_{n m}\end{array}\right)$ be an $n \times m$ matrix; if $a_{i j}=x+y I_{1}+z I_{2} \in R_{2}(I)$, then it is called an refined neutrosophic matrix, where $R_{2}(I)$ is an refined neutrosophic field.

Example 1. $X=\left(\begin{array}{cc}I_{1} & I_{1}+I_{2} \\ 3-I_{1} & 2 I_{2}\end{array}\right)$ is a $2 \times 2$ refined neu-
trosophic matrix.

Remark 2 (addition and multiplication, ring structure)

(a) If $A$ is an $m \times n$ matrix, then it can be represented as an element of the refined neutrosophic ring of matrices such as the following: $A=B+C I_{1}+D I_{2}$, where $D, B$, and $C$ are classical matrices with elements from ring $R$ and from size $m \times n$.

For example, $A=\left(\begin{array}{cc}2+I_{1}+3 I_{2} & 1-I_{1}-I_{2} \\ 3+4 I_{2} & 1+I_{1}\end{array}\right)=$ $\left(\begin{array}{ll}2 & 1 \\ 3 & 1\end{array}\right)+\left(\begin{array}{cc}1 & -1 \\ 0 & 1\end{array}\right) I_{1}+\left(\begin{array}{cc}3 & -1 \\ 4 & 0\end{array}\right) I_{2}$

(b) The addition operation can be defined by using the representation in Remark 2 as follows:

$$
\left(A+B I_{1}+C I_{2}\right)+\left(X+Y I_{1}+Z I_{2}\right)=(A+X)+(B+Y) I_{1}+(C+Z) I_{2}
$$

(c) Multiplication can be defined by using the same representation as a special case of multiplication on refined neutrosophic rings as follows:

$$
\left(A+B I_{1}+C I_{2}\right)\left(X+Y I_{1}+Z I_{2}\right)=(A X)+(A Y+B X+B Y+B Z+C Y) I_{1}+(A Z+C Z+C X) I_{2}
$$

This method of multiplication is exactly equivalent to the normal multiplication between matrices, but it is easier to deal with in this way.

Example 2. Let $X=\left(\begin{array}{cc}I_{1} & I_{1}+I_{2} \\ 3-I_{1} & 2 I_{2}\end{array}\right)$ and $Y=\left(\begin{array}{cc}-1 & I_{1} \\ 1+I_{2} & 3 I_{1}\end{array}\right)$ be two refined neutrosophic matrices over the refined neutrosophic field of reals. We have

$$
\begin{aligned}
& \text { (a) } X=A+B I_{1}+C I_{2} ; A=\left(\begin{array}{ll}
0 & 0 \\
3 & 0
\end{array}\right), \quad B=\left(\begin{array}{cc}
1 & 1 \\
-1 & 0
\end{array}\right) \text {, } \\
& \text { and } C=\left(\begin{array}{ll}
0 & 1 \\
0 & 2
\end{array}\right) \text {. }
\end{aligned}
$$

(b) $Y=M+N I_{1}+S I_{2} ; M=\left(\begin{array}{cc}-1 & 0 \\ 1 & 0\end{array}\right), \quad N=\left(\begin{array}{ll}0 & 1 \\ 0 & 3\end{array}\right)$,
and $S=\left(\begin{array}{ll}0 & 0 \\ 1 & 0\end{array}\right)$.

(c) $X+Y=\left(\begin{array}{cc}-1+I_{1} & 2 I_{1}+I_{2} \\ 4-I_{1}+I_{2} & 3 I_{1}+2 I_{2}\end{array}\right)$.

(d) $X Y=\left(\begin{array}{cc}-I_{1}+\left(I_{1}+I_{2}\right)\left(1+I_{2}\right) & I_{1} I_{1}+\left(I_{1}+I_{2}\right)\left(3 I_{1}\right) \\ -3+I_{1}+\left(2 I_{2}\right)\left(1+I_{2}\right)\left(3-I_{1}\right)\left(I_{1}\right)+\left(2 I_{2}\right)\left(3 I_{1}\right)\end{array}\right)=$

$$
\left(\begin{array}{cc}
I_{1}+2 I_{2} & 7 I_{1} \\
-3+I_{1}+4 I_{2} & 8 I_{1}
\end{array}\right)
$$

(e) If we compute the multiplication using the representation of Remark 2, we get 


$$
\begin{aligned}
& A M=\left(\begin{array}{cc}
0 & 0 \\
-3 & 0
\end{array}\right) \text {, } \\
& A N=\left(\begin{array}{ll}
0 & 0 \\
0 & 3
\end{array}\right) \text {, } \\
& B M=\left(\begin{array}{ll}
0 & 0 \\
1 & 0
\end{array}\right), \\
& B N=\left(\begin{array}{cc}
0 & 4 \\
0 & -1
\end{array}\right) \text {, } \\
& B S=\left(\begin{array}{ll}
1 & 0 \\
0 & 0
\end{array}\right) \text {, } \\
& C N=\left(\begin{array}{ll}
0 & 3 \\
0 & 6
\end{array}\right) \text {, } \\
& A S=\left(\begin{array}{ll}
0 & 0 \\
0 & 0
\end{array}\right), \\
& C S=\left(\begin{array}{ll}
1 & 0 \\
2 & 0
\end{array}\right) \text {, } \\
& C M=\left(\begin{array}{ll}
1 & 0 \\
2 & 0
\end{array}\right) .
\end{aligned}
$$

Hence, $\quad X Y=A M+I_{1}(A N+B N+B M+B S+C N)$

$+I_{2}(A S+C S+C M)=\left(\begin{array}{cc}0 & 0 \\ -3 & 0\end{array}\right)+I_{1}\left(\begin{array}{ll}1 & 7 \\ 1 & 8\end{array}\right)+I_{2}\left(\begin{array}{ll}2 & 0 \\ 4 & 0\end{array}\right)$

$=\left(\begin{array}{cc}I_{1}+2 I_{2} & 7 I_{1} \\ -3+I_{1}+4 I_{2} & 8 I_{1}\end{array}\right)$.

Theorem 3. The set of all square $n \times n$ refined neutrosophic matrices together makes a ring.

Proof. The proof holds directly from the definition of $n$ refined neutrosophic rings by taking $n=2$.

Remark 3. The identity with respect to multiplication is the normal unitary matrix.

Definition 7. Let $A$ be a square $n \times n$ refined neutrosophic matrix; then, it is called invertible if there exists a refined square $n \times n$ neutrosophic matrix $B$ such that $A B=U_{n \times n}$, where $U_{n \times n}$ is the unitary classical matrix.
Theorem 5. Let $X=A+B I_{1}+C I_{2}$ be a square $n \times n$ refined neutrosophic matrix; then, it is invertible if and only if $A, A+$ $C$, and $A+B+C$ are invertible. The inverse of $X$ is $X^{-1}=$ $A^{-1}+\left((A+B+C)^{-1}-(A+C)\right)^{-1} I_{1}+\left((A+C)^{-1}-A^{-1}\right) I_{2}$.

Proof. The proof holds as a special case of invertible elements in refined neutrosophic rings [8].

Definition 8. We define the determinant of a square $n \times n$ refined neutrosophic matrix as $\operatorname{det} X=\operatorname{det} A+[\operatorname{det}(A+B+$ $C)-\operatorname{det}(A+C)] I_{1}+[\operatorname{det}(A+C)-\operatorname{det} A] I_{2}$.

This definition is supported by the condition of invertibility.

Theorem 6. Let $X=A+B I_{1}+C I_{2}$ be a square $n \times n$ refined neutrosophic matrix; we have the following:

(a) $X$ is invertible if and only if $\operatorname{det} X \neq 0$

(b) If $Y=M+N I_{1}+S I_{2}$ is a square $n \times n$ refined neutrosophic matrix, then $\operatorname{det} X Y=\operatorname{det} X \operatorname{det} Y$

(c) $\operatorname{det} X^{-1}=(\operatorname{det} X)^{-1}$

Proof

(a) If $\operatorname{det} X \neq 0$, this will be equivalent to $\operatorname{det} A \neq 0, \operatorname{det}(A+C) \neq 0, \quad$ and $\operatorname{det}(A+B+C) \neq 0$, i.e., $A, A+C$, and $A+B+C$ are invertible; thus, $X$ is invertible according to Theorem 5 .

(b) $X Y=A M+I_{1}[(A+B+C)(M+N+S)-(A+C)$ $(M+S)]+I \quad 2[(A+C)(M+S)-A M]$. Hence, $\operatorname{det} X Y=\operatorname{det}(A M)+I_{1}[\operatorname{det}((A+B+C) \quad(M+N+$ $S))]+I_{2}[\operatorname{det}((A+C)(M+S))]=\operatorname{det} A \operatorname{det} M+I_{1}$ $[\operatorname{det}(A+B+C) \operatorname{det}(M+N+S)]+I \quad 2[\operatorname{det}(A+C)$ $\operatorname{det}(M+S)]=\left(\operatorname{det} A+I_{1}[\operatorname{det}(A+B+C)-\operatorname{det}(A+\right.$ $\left.C)]+I_{2}[\operatorname{det}(A+C)-\operatorname{det} A]\right) \quad\left(\operatorname{det} M+I_{1}[\operatorname{det}(M+\right.$ $\left.N+S)-\operatorname{det}(M+S)]+I_{2}[\operatorname{det}(M+S)-\operatorname{det} M]\right)=$ $\operatorname{det} X \operatorname{det} Y$.

(c) It holds directly from (b).

Theorem 7. Let $X=A+B I_{1}+C I_{2}$ be a square $n \times n$ refined neutrosophic matrix; we have the following:

(a) $X$ is nilpotent if and only if $A, A+C$, and $A+B+C$ are nilpotent

(b) $X$ is idempotent if and only if $A, A+C$, and $A+B+$ $C$ are idempotent

Proof

(a) First of all, we will prove that $X^{r}=A^{r}+I_{1}[(A+B+$ $\left.C)^{r}-(A+C)^{r}\right]+I_{2}\left[(A+C)^{r}-A^{r}\right]$.

We use the induction, for $r=1$, it is clear. Suppose that it is true for $r=k$, we prove it for $k+1$. 


$$
\begin{aligned}
X^{k+1}= & X^{k} X=\left(A^{k}+I_{1}\left[(A+B+C)^{k}-(A+C)^{k}\right]+I_{2}\left[(A+C)^{k}-A^{k}\right]\right)\left(A+B I_{1}+C I_{2}\right) \\
= & A^{k+1}+I_{1}\left[A^{k} B+(A+B+C)^{k} A+(A+B+C)^{k} B+(A+B+C)^{k} C-(A+C)^{k} A-(A+C)^{k} B\right. \\
& \left.-(A+C)^{k} C+(A+C)^{k} B-A^{k} B\right]+I_{2}\left[A^{k} C+(A+C)^{k} C-A^{k} C+(A+C)^{k} A-A^{k} A\right] \\
= & A^{k+1}+I_{1}\left[(A+B+C)^{k+1}-(A+C)^{k+1}\right]+I_{2}\left[(A+C)^{k+1}-A^{k+1}\right] .
\end{aligned}
$$

$X$ is nilpotent if there is a positive integer $r$ such that $X^{r}=O_{n \times n}$. This is equivalent to

$$
\begin{array}{r}
A^{r}=(A+C)^{r}=(A+B+C)^{r}=O_{n \times n}, \\
\text { which implies the proof. }
\end{array}
$$

(b) The proof is similar to (a).

Example 4. Consider the following refined neutrosophic matrix $A=\left(\begin{array}{cc}2+I_{1}+3 I_{2} & 1-I_{1}-I_{2} \\ 3+4 I_{2} & 1+I_{1}\end{array}\right)$; we have the
following:

(a) $A=\left(\begin{array}{cc}2+I_{1}+3 I_{2} & 1-I_{1}-I_{2} \\ 3+4 I_{2} & 1+I_{1}\end{array}\right)=\left(\begin{array}{ll}2 & 1 \\ 3 & 1\end{array}\right)+\left(\begin{array}{cc}1 & -1 \\ 0 & 1\end{array}\right)$ $I_{1}+\left(\begin{array}{cc}3 & -1 \\ 4 & 0\end{array}\right) I_{2}$.

$B=\left(\begin{array}{ll}2 & 1 \\ 3 & 1\end{array}\right), \quad C=\left(\begin{array}{cc}1 & -1 \\ 0 & 1\end{array}\right)$, and $D=\left(\begin{array}{cc}3 & -1 \\ 4 & 0\end{array}\right)$. $B+D=\left(\begin{array}{ll}5 & 0 \\ 7 & 1\end{array}\right)$, and $B+C+D=\left(\begin{array}{cc}6 & -1 \\ 7 & 2\end{array}\right)$.

(b) $B^{-1}=\left(\begin{array}{cc}-1 & 1 \\ 3 & -2\end{array}\right), \quad(B+D)^{-1}=\left(\begin{array}{cc}1 / 5 & 0 \\ -7 / 5 & 1\end{array}\right), \quad$ and $(B+C+D)^{-1}=\left(\begin{array}{cc}2 / 19 & 1 / 19 \\ -7 / 19 & 6 / 19\end{array}\right)$.

(c) $A^{-1}=B^{-1}+I_{1}\left[(B+C+D)^{-1}-(B+D)^{-1}\right]+I_{2}$ $\left[(B+D)^{-1}-B^{-1}\right]=\left(\begin{array}{cc}-1 & 1 \\ 3 & -2\end{array}\right)+I_{1}\left(\begin{array}{cc}-9 / 95 & 1 / 19 \\ 98 / 95 & -13 / 19\end{array}\right)+$ $I_{2}\left(\begin{array}{cc}6 / 5 & -1 \\ -22 / 5 & 3\end{array}\right)=\left(\begin{array}{cc}-1-(9 / 95) I_{1}+(6 / 5) I_{2} & 1+(1 / 19) I_{1}-I_{2} \\ 3+(98 / 95) I_{1}-(22 / 5) I_{2} & -2-(13 / 19) I_{1}+3 I_{2}\end{array}\right)$. It is easy to find that $A^{-1} A=A A^{-1}=\left(\begin{array}{ll}1 & 0 \\ 0 & 1\end{array}\right)$.

(d) $\operatorname{det} B=-1, \operatorname{det}(B+D)=5, \operatorname{det}(B+C+D)=19$, $\operatorname{det} A=-1+I_{1}[19-5]+I_{2}[5-(-1)]=-1+14 I_{1}$ $+6 I_{2}$.

If we compute the determinant of $A$ by using the classical way, we will get the same result.

Now, we illustrate an example to clarify the application of refined neutrosophic matrices in solving refined neutrosophic algebraic equations defined in [17].

Example 5. Consider the following system of refined neutrosophic linear equations:

$$
\begin{aligned}
\left(2+I_{1}+3 I_{2}\right) X+\left(1-I_{1}-I_{2}\right) Y & =-I_{1}(*), \\
\left(3+4 I_{2}\right) X+\left(1+I_{1}\right) Y & =I_{2}(* *) .
\end{aligned}
$$

The corresponding refined neutrosophic matrix is $A=\left(\begin{array}{cc}2+I_{1}+3 I_{2} & 1-I_{1}-I_{2} \\ 3+4 I_{2} & 1+I_{1}\end{array}\right)$.

Since $A$ is invertible, we get the solution of the previous system by computing the product:

$$
\begin{aligned}
A^{-1}\left(\begin{array}{c}
-I_{1} \\
I_{2}
\end{array}\right) & =\left(\begin{array}{cc}
-1-\frac{9}{95} I_{1}+\frac{6}{5} I_{2} & 1+\frac{1}{19} I_{1}-I_{2} \\
3+\frac{98}{95} I_{1}-\frac{22}{5} I_{2} & -2-\frac{13}{19} I_{1}+3 I_{2}
\end{array}\right)\left(\begin{array}{c}
-I_{1} \\
I_{2}
\end{array}\right) \\
& =\left(\begin{array}{c}
I_{1}\left[1+\frac{9}{95}-\frac{6}{5}+\frac{1}{19}\right] \\
I_{1}\left[-3-\frac{98}{95}+\frac{22}{5}-\frac{13}{19}\right]+I_{2}[-2+3]
\end{array}\right) \\
& =\left(\begin{array}{c}
-I_{1}(1 / 19) \\
-(6 / 19) I_{1}+I_{2}
\end{array}\right) . \text { Thus, } X=-\frac{1}{19} I_{1}, \\
Y & =-\frac{6}{19} I_{1}+I_{2} .
\end{aligned}
$$

\section{Conclusion}

In this paper, we have used the concept of refined neutrosophic set to build the corresponding refined neutrosophic matrix. On the contrary, many interesting properties have been studied and proved such as idempotency, nilpotency, determinants, and invertibility of these matrices.

Also, a direct application of these matrices was proposed in solving refined neutrosophic equations.

As a future research direction, we aim to study the diagonalization properties with eigenvectors of these matrices.

\section{Data Availability}

No data were used to support this study.

\section{Conflicts of Interest}

The author declares that there are no conflicts of interest. 


\section{References}

[1] M. Abdel-Basset, A. Gamal, L. H. Son, and F. Smarandache, "A bipolar neutrosophic multi criteria decision making framework for professional selection," Applied Sciences, vol. 10, no. 4, p. 1202, 2020.

[2] H. Sankari and M. Abobala, "Neutrosophic linear diophantine equations with two variables," Neutrosophic Sets and Systems, vol. 38, 2020.

[3] M. Abobala, "Foundations of neutrosophic number theory," Neutrosophic Sets and Systems, vol. 39, 2021.

[4] M. Abobala, "A study of AH-substructures in n-refined neutrosophic vector spaces," International Journal of Neutrosophic Science, vol. 9, pp. 74-85, 2020.

[5] M. Abobala and R. Alhamido, "AH-substructures in neutrosophic modules," International Journal of Neutrosophic Science, vol. 7, pp. 79-86, 2020.

[6] A. A. A. Agboola, "On refined neutrosophic algebraic structures," Neutrosophic Sets and Systems, vol. 10, pp. 99-101, 2015.

[7] E. O. Adeleke, A. A. A. Agboola, and F. Smarandache, "Refined neutrosophic rings II," International Journal of Neutrosophic Science, vol. 2, pp. 89-94, 2020.

[8] M. Abobala, "On some special elements in neutrosophic rings and refined neutrosophic rings," Journal of New Theory, vol. 33, 2020.

[9] H. Sankari and M. Abobala, "AH-homomorphisms in neutrosophic rings and refined neutrosophic rings," Neutrosophic Sets and Systems, vol. 38, 2020.

[10] M. Abobala and A. Hatip, "AH-substructures in strong refined neutrosophic modules," International Journal of Neutrosophic Science, vol. 9, pp. 110-116, 2020.

[11] M. Abobala, "Semi homomorphisms and algebraic relations between strong refined neutrosophic modules and strong neutrosophic modules," Neutrosophic Sets and Systems, vol. 39, 2021.

[12] F. Smarandache and M. Abobala, "n-Refined neutrosophic rings," International Journal of Neutrosophic Science, vol. 6, pp. 83-90, 2020.

[13] F. Smarandache and M. Abobala, " $n$-Refined neutrosophic vector spaces," International Journal of Neutrosophic Science, vol. 7, pp. 47-54, 2020.

[14] H. Sankari and M. Abobala, "n-Refined neutrosophic modules," Neutrosophic Sets and Systems, vol. 36, 2020.

[15] M. Dhar, S. Broumi, and F. Smarandache, "A note on square neutrosophic fuzzy matrices," Neutrosophic Sets and Systems, vol. 3, pp. 37-41, 2014.

[16] H. Khaled, A. Younus, and A. Mohammad, "The rectangle neutrosophic fuzzy matrices," Faculty of Education Journal, vol. 15, 2019 (Arabic version).

[17] M. Abobala, "On some neutrosophic algebraic equations," Journal of New Theory, vol. 33, 2020. 